\title{
Numerical Analysis of Pool Fires in Oil Trunkline Networks
}

\author{
Vadim E. Seleznev \\ Physical \& Technical Center, LLC, P.O. Box 236 Sarov, Nizhny Novgorod Region 607190, Russia \\ Correspondence should be addressed to Vadim E. Seleznev, sve@ptc.sar.ru
}

Received 22 April 2010; Revised 2 July 2010; Accepted 28 July 2010

Academic Editor: Yiguang Ju

Copyright ( 2010 Vadim E. Seleznev. This is an open access article distributed under the Creative Commons Attribution License, which permits unrestricted use, distribution, and reproduction in any medium, provided the original work is properly cited.

This paper presents a method for numerical evaluation of parameters of flammable liquid pool fires caused by storage tank or trunkline failures. The method may be useful for specialists working in oil, gas, and chemical industries. It was successfully applied in fire safety analysis of Russian gas and oil processing facilities.

\section{Statement of the Problem}

This paper is a logical sequel to the description of practical methods for mathematical simulation of fires at gas and oil facilities, whose methods were initially described in [1]. As is well known, combustion of liquid fuel spilled on the terrain adjacent to the region of trunkline (or storage tank) rupture takes place as combustion of a stream of its vapor in the air. One of the main tasks of the considered approach practical application is to obtain significant upper estimate of potential or analysis of actual consequences of heat damage for facilities adjacent to pool fire site at the rupture region of trunklines transporting combustible fluids. At that, it is necessary to take into consideration not only intensity, but also duration of fire.

The stream of vapor in the flame is maintained by continuous evaporation. The rate of evaporation is determined by the rate of the heat flow coming from the flame to the liquid fuel. Oxygen required for combustion comes to the reaction zone from the ambient air. The flame of the combusting liquid fuel can be treated as a diffusion flame. Accordingly, for the purpose of simulation, it is advisable to consider liquid fuel combustion as a specific case of liquid evaporation accompanied by combustion of nonmingled gases (vapor and oxidant) [2].

High-accuracy simulation of pool fires is an extremely difficult task because of the complex and varied nature of the physical and chemical processes involved. As such processes, we can consider formation of a homometric layer in liquid fuel, boiling and evaporation of the fuel, its splashing, ignition, and combustion of liquid fuel vapors.

Mechanisms of these processes may vary substantially, according to the type of fuel, the condition and type of the soil at the accident site, weather conditions, and so forth. This paper describes a method for numerical simulation of combustion of liquid fuels transmitted through trunklines and/or stored in tanks, with the aim of making estimative calculations of parameters of actual or potential pool fires.

Solving the problem, we proceed from an assumption that the liquid fuel spillage occurred on a porous terrain. Parameters of the spillage spot on the terrain are assumed to be known. On a first approximation, simulation conventionally disregards the phase of fuel warmup. The chemical reactions of combustion are assumed to be infinitely rapid.

\section{Simulation of a Fuel Evaporation Phase}

The liquid fuel evaporates into a gas phase and a combustible mixture develops. In estimative simulation of fuel evaporation, the following simplifications and assumptions are admitted (see [2]):

(i) the fuel and the environment during evaporation are assumed to be in a quasistationary state;

(ii) the processes of heat and mass transfer are assumed to be identical (the Lewis number being equal to one); 
(iii) with respect to the mixture of atmospheric air and fuel vapors, we assume that the heat conductivity coefficient $\lambda$ and the product of the mixture density $\rho$ and the coefficient of fuel vapors binary diffusion in the atmospheric air $D_{\text {vap }}$ are constant and not dependent on the temperature $T$;

(iv) the specific heat of fuel vapors $c_{p}^{\text {vap }}$ is assumed to be constant and not dependent on temperature;

(v) fuel vapors are assumed to be diffusing in the still air along the vertical coordinate axis $\mathrm{Oz}$ directed away from the center of the Earth;

(vi) the magnitude of the diffusion mass flow $(J=\rho w)$ has finite value only with respect to fuel vapors, that is, $J=J_{\text {vap }}$, where $w$ is the projection of an average velocity of the center of mass of the fuel vapors and atmospheric air mixture onto the coordinate axis $\mathrm{Oz}$ ( $w$ being the only nonzero projection of the velocity), usually in the context of fluid mechanics, such a flow is called convective whereas, in this case, its name has been changed in accordance with the physics of evaporation processes and the recommendations contained in [2];

(vii) the shear stresses work and kinetic energy are negligible;

(viii) there are no outside sources of energy in the fuel evaporation zone;

(ix) the air is not dissolving in the fuel;

(x) no chemical reactions take place during evaporation.

For this reason, we will obtain evaluation of the diffusion mass flow of fuel vapors into the combustion zone in a different way. Let us write down, subject to the above assumptions and simplifications, a modification of the following well-known energy equation [3]:

$$
-\lambda \frac{d^{2} T}{d z^{2}}+\rho w c_{p}^{\operatorname{vap}} \frac{d T}{d z}=0 .
$$

The first integration (1) produces

$$
\begin{aligned}
& \lambda \frac{d T}{d z}-\rho w c_{p}^{\mathrm{vap}} T=-Q_{0}-\rho w c_{p}^{\mathrm{vap}} T_{0} \\
& \text { or } \frac{d T}{d z}=\frac{1}{\lambda}\left[\rho w c_{p}^{\mathrm{vap}}\left(T-T_{0}\right)-Q_{0}\right] .
\end{aligned}
$$

After the second integration, we obtain the following:

$$
\ln \left[T-T_{0}-\frac{Q_{0}}{\rho w c_{p}^{\mathrm{vap}}}\right]=\frac{\rho w c_{p}^{\mathrm{vap}} z}{\lambda}+\text { const. }
$$

Substitution of boundary conditions (if $z=0: T=T_{0}$ ) and (if $z=L: T=T_{L}$ ) allows us to write down

$$
\begin{gathered}
\text { const }=\ln \left[-\frac{Q_{0}}{\rho w c_{p}^{\mathrm{vap}}}\right], \\
\ln \left[1-\frac{c_{p}^{\mathrm{vap}}\left(T_{L}-T_{0}\right)}{Q_{0} /(\rho w)}\right]=\frac{\rho w c_{p}^{\mathrm{vap}} L}{\lambda} .
\end{gathered}
$$

It was noted before that on a first approximation we disregard the processes of fuel warmup. Then, we can assume that, in the simulated pool fire, from the moment the fire starts and till the moment the spilled liquid fuel burns out, the liquid fuel free surface has the temperature equal to the boiling temperature $T_{\text {boil }}$ of this liquid fuel. This assumption is quite admissible, since, in the fuel warmup phase, energy is transferred to its surface layer until it reaches the boiling temperature [4]. The heat delivered to the liquid fuel from the ground and other flame-unrelated sources is disregarded in this case. Then, it possible to write that $\left(Q_{0}=-\rho w q_{0}\right)[2]$. As a result, we obtain evaluation of the diffusion mass flow of fuel vapors into the combustion zone in the following form:

$$
J_{\text {vap }}=\rho w=\frac{\lambda}{L c_{p}^{\text {vap }}} \ln \left[1+\frac{c_{p}^{\text {vap }}\left(T_{L}-T_{\text {boil }}\right)}{q_{0}}\right]
$$

In the process of numerical solution of a problem by mesh methods, the temperature $T_{L}$ is generally associated with the time-variable temperature of the mixture of liquid fuel vapors and the air at the spatial computation mesh nods nearest to fuel surface on the gas phase side. In the computation, the heat conductivity coefficient $\lambda$ in (5) is substituted with the coefficient of effective heat conductivity computable by the reference data and methods described, for instance, in [3]. Values $c_{p}^{\text {vap }}$ and $q_{0}$ are experimental reference data.

In pool fire simulation, formula (5) allows to make an upper evaluation of behavior parameters of the source of flammable vapors. Given the functional relationship of the diffusion mass flow of its vapors (5) is known, it is not difficult to estimate the time when the spilled liquid will be burned out. This estimate is required to forecast the duration of fire.

\section{Simulation of a Phase of Liquid Fuel Vapors Combustion}

At this stage, we make numerical analysis of volatile vapors combustion parameters in the gas phase, treating it as a nonpremingled gaseous mixture and using the approaches set forth in [1]. Here, we use the turbulent eddy breakup model proposed by Spalding [2]. Subject to the above, simulation of the combustion phase (on the assumption of a single-stage irreversible index-recorded reaction between fuel vapors and the oxidant) is reduced to numerical analysis of the following set of equations $[1,5]$ :

$$
\begin{gathered}
\frac{D \rho}{D t}+\rho(\vec{\nabla} \cdot \overrightarrow{\mathbf{V}})=0, \\
\rho \frac{D Y_{\text {vap }}}{D t}=\vec{\nabla} \cdot\left(\frac{\mu+\mu_{T}}{\mathrm{Sc}_{\text {vap }}} \cdot \vec{\nabla} Y_{\text {vap }}\right)-\omega_{\text {vap }}, \\
\rho \frac{D Y_{\text {air }}}{D t}=\vec{\nabla} \cdot\left(\frac{\mu+\mu_{T}}{\mathrm{Sc}_{\text {air }}} \cdot \vec{\nabla} Y_{\text {air }}\right)-\xi_{\text {air }}^{\text {mass }} \omega_{\text {vap }}, \\
Y_{\text {prod }}=1-Y_{\text {vap }}-Y_{\text {air }},
\end{gathered}
$$




$$
\begin{aligned}
& \omega_{\text {vap }}=\rho A \frac{\varepsilon}{K} \cdot \min \left(Y_{\text {vap }}, \frac{Y_{\text {air }}}{\xi_{\text {air }}}, \frac{B Y_{\text {prod }}}{1+\xi_{\text {air }}^{\text {mass }}}\right) \text {, } \\
& \rho \frac{D \overrightarrow{\mathbf{V}}}{D t}=\left(\rho-\rho_{\text {atm }}\right) \overrightarrow{\mathbf{g}}-\vec{\nabla} P \\
& +\vec{\nabla} \cdot\left(\frac{\mu+\mu_{T}}{\mu} \tau\right)-\frac{2}{3} \vec{\nabla}(\rho K), \\
& \rho \frac{D H}{D t}=\frac{\partial P}{\partial t}+Q_{\mathrm{comb}} \omega_{\mathrm{vap}}-S_{\mathrm{rad}}+\frac{\partial Q}{\partial t}+\rho \overrightarrow{\mathbf{g}} \cdot \overrightarrow{\mathbf{V}} \\
& +\vec{\nabla} \cdot\left(\left(\lambda+\lambda_{T}\right) \cdot \vec{\nabla} T+\frac{\mu+\mu_{T}}{\mu} \boldsymbol{\tau} \cdot \overrightarrow{\mathbf{V}}-\frac{2}{3} \rho K \overrightarrow{\mathbf{V}}\right) \\
& +\sum_{m=1}^{N^{*}} \vec{\nabla} \cdot\left[e_{m} \frac{\mu+\mu_{T}}{\mathrm{Sc}_{m}} \cdot \vec{\nabla} Y_{m}\right] \\
& \rho \frac{D K}{D t}=\vec{\nabla} \cdot\left[\frac{\mu+\mu_{T}}{\operatorname{Pr}_{K}} \cdot \vec{\nabla} K\right]+G-\rho \varepsilon-\frac{\mu_{T}}{\rho \operatorname{Pr}_{T}}(\overrightarrow{\mathbf{g}} \cdot \vec{\nabla} \rho), \\
& \rho \frac{D \varepsilon}{D t}=\vec{\nabla} \cdot\left[\frac{\mu+\mu_{T}}{\operatorname{Pr}_{\varepsilon}} \cdot \vec{\nabla} \varepsilon\right] \\
& +\frac{\varepsilon}{K}\left(C_{2} G-C_{3} \rho \varepsilon-C_{1} \frac{\mu_{T}}{\rho \operatorname{Pr}_{T}}(\overrightarrow{\mathbf{g}} \cdot \vec{\nabla} \rho)\right), \\
& S_{\mathrm{rad}}=\sum_{j=1}^{L^{*}} \varphi_{j}\left(Y_{j}\right) \cdot \int_{\nu=0}^{\infty} \vec{\nabla} \cdot \overrightarrow{\mathbf{q}}_{\nu, j}^{r} d \nu, \quad \text { where } \\
& \vec{\nabla} \cdot \overrightarrow{\mathbf{q}}_{\nu, j}^{r}=\chi_{\nu, j}\left(4 \pi I_{\nu b}(T)-\int_{4 \cdot \pi} I_{\nu, j}(s, \overrightarrow{\boldsymbol{\theta}}, t) d \vartheta\right), \\
& \frac{1}{c_{\mathrm{rad}}} \frac{\partial I_{\nu, j}(s, \overrightarrow{\boldsymbol{\theta}}, t)}{\partial t}+\overrightarrow{\boldsymbol{\theta}} \cdot \vec{\nabla} I_{\nu, j}(s, \overrightarrow{\boldsymbol{\theta}}, t) \\
& +\left(\chi_{v, j}+\beta_{v, j}\right) \cdot I_{v, j}(s, \overrightarrow{\boldsymbol{\theta}}, t) \\
& =\chi_{\nu, j} I_{\nu b}(T)+\frac{\beta_{\nu, j}}{4 \cdot \pi} \\
& \cdot \int_{4 \cdot \pi} \gamma_{\nu, j}\left(\overrightarrow{\boldsymbol{\theta}}, \overrightarrow{\boldsymbol{\theta}^{\prime}}\right) \cdot I_{\nu, j}\left(s, \overrightarrow{\boldsymbol{\theta}^{\prime}}, t\right) d \vartheta^{\prime}, \\
& j=\overline{1, L^{*}} \\
& P=\rho R T+\rho_{\mathrm{atm}} g\left(x_{3}-x_{3,0}\right) \text {, } \\
& R=R_{0} \sum_{m=1}^{N^{*}} \frac{Y_{m}}{M_{m}} \\
& H=C_{p} T+\frac{\overrightarrow{\mathbf{V}} \cdot \overrightarrow{\mathbf{V}}}{2}, \\
& \alpha=\alpha_{\text {mix }}=\sum_{m=1}^{N^{*}} f_{\alpha}\left(Y_{m}, \alpha_{m}\right), \quad \text { where } \alpha \equiv \mu, \lambda, C_{p}, C_{v} \text {, }
\end{aligned}
$$

$$
\begin{gathered}
\mu=\left(\frac{T}{273.15}\right)^{3 / 2} \cdot \frac{273.15+C_{S}}{T+C_{S}} \mu_{0}, \\
\mu_{T}=\frac{C_{\mu} \rho K^{2}}{\varepsilon}, \quad \lambda_{T}=\frac{C_{p} \mu_{T}}{\operatorname{Pr}_{T}}, \\
\tau_{i j}=\mu\left[\left(\frac{\partial u_{i}}{\partial x_{j}}+\frac{\partial u_{j}}{\partial x_{i}}\right)-\frac{2}{3} \delta_{i j} \frac{\partial u_{k}}{\partial x_{k}}\right], \\
G=\mu_{T}\left\{\frac{1}{2}\left(\frac{\partial u_{i}}{\partial x_{j}}+\frac{\partial u_{j}}{\partial x_{i}}\right)^{2}-\frac{2}{3}\left(\frac{\partial u_{k}}{\partial x_{k}}\right)^{2}\right\}-\frac{2}{3} \rho K \frac{\partial u_{k}}{\partial x_{k}},
\end{gathered}
$$

where $D(\cdots) / D t \equiv \partial(\cdots) / \partial t+\overrightarrow{\mathbf{V}} \cdot \vec{\nabla}(\cdots)$ is the substantial derivative of the scalar function; the notation of the substantial derivative of the scalar function means substantial differentiation of vector function components; the subscript atm means that the parameter belongs to undisturbed atmosphere parameters; $N^{*}=3$ in the particular case; $\partial Q / \partial t$ is specified per-unit-volume velocity of heat radiation of outside sources (the specified perunit-volume capacity of the heat sources); the subscript $K$ means that the value of the Prandtl number is specified expressly for the equation of turbulent energy (10); subscript $\varepsilon$ is for the equation of turbulence dissipation (11); subscript $T$, when placed beside parameters, means "turbulent".

Equations (6)-(7) in the model given above describe a mass conservation law for multicomponent mixture which contains fuel vapors and air as an oxidant, under conditions of intensive exothermal chemical reaction development. The velocity of reaction is estimated according to the law (7). Equations (8) and (9) describe a momentum conservation law and energy conservation law, respectively. In this case, Reynolds set of equations is closed with the wellknown $(k-\varepsilon)$-turbulence model (10), (11). To close the system, equations of state (14) and auxiliary formulae (15) are used.

It should be noted here that the Reynolds equations (6)(15) use averaged parameters of the gaseous mixture flow. At that, the velocity components and the thermal variables $(H, h, T)$ are averaged according to Favre (i.e., using the mixture density as a weighting function), and the density and pressure-according to the classical Reynolds averaging [6]. Such a scheme of averaging is effective as applied to simulation of flows of a compressible gaseous mixture. It should be noted here that, to enhance the accuracy of simulation, it would be well to use the equation written for averaging of the reaction velocity rather than (7). The mechanism of such averaging using a statistical approach is set forth in [4]. The use of such an approach results in the incorporation of a new probability-density function transfer equation into the set (6)-(15), which significantly complicates solution of practical problems (at the present stage of development and availability of computer machines, this renders solution of industrial problems facing fuel and 
power facilities unpractical). For this reason, in this case we have to agree to a lower adequacy of simulation of combustion processes and use (7).

The set of equations (6)-(15) is supplemented by relevant boundary conditions depending on the specific statement of the problem. In this connection, (5) is used to estimate fuel vapors flow towards the combustion area. Additional recommendations on the appropriate formation of boundary conditions are given in [5]. This set of equations is solved by the finite volume method or the finite element method [7].

Energy equation (9) includes radiation source term $S_{\text {rad }}$ (see also (12)). The most adequate and consistent procedure for numerical evaluation of a contribution by the radiation energy transfer to the plume parameters variation amounts (i.e., estimation $S_{\text {rad }}$ ) to solution of integrodifferential equations of radiant energy transfer (13) for each of the gaseous combustion products. It should be noted here that, in (13) being considered, we can disregard the first term compared to other terms, because of the greater velocity of the radiation propagation. In numerical analysis of fire parameters for the integration of functions containing the spectral indicatrix of dissipation $\gamma_{\nu, j}\left(\overrightarrow{\boldsymbol{\theta}}, \overrightarrow{\boldsymbol{\theta}^{\prime}}\right)$, we use its Legendre polynomial expansion. At that, solution of (13) is carried out by the Monte-Carlo method, the modified method of average flows, or the method of a weighted sum of grey gases [8]. This particular method has been chosen because of its potential implementation for computer hardware available for wide range of pipeline transport experts. At the same time to solve (13), it is possible to use a well-known method of average flows.

Ignition of the air-and-fuel mixture is simulated using a thermal model of ignition, whose application algorithm is described in details in [5]. The equations (6)-(15) can be easily extended to the case of analysis of multistage chemical reactions subject to combustion products dissociation involving energy consumption.

\section{On the Question of Evaluating Pool Fire Duration}

In analysis of the mechanisms of pool fire extinction, it is required to evaluate the quantity of liquid fuel infiltrated into the soil and the geometry of the zone of its infiltration into the soil layer. It is important for correct estimation of the simulated pool fire duration. To solve the problem posed, we use numerical analysis of parameters of isothermal single-phase gravity filtration of noncompressible viscous chemically inert liquid into a nondeformable layer of soil. The viscosity of the liquid is assumed to be constant in this case. Taking into account the analysis of actual emergency situations, the duration of the spilling process is assumed to be much shorter than that of the filtration process. Such an assumption allows the simulation to separate the processes of spilling and filtration, which enables us to omit solution of the conjugate problem.

In case of the isothermal single-phase gravity filtration of viscous liquid into an isotropic porous medium, the defining equations are set in the form of [9]

$$
\begin{gathered}
\frac{\partial\left(m_{\text {soil }} \rho_{l}\right)}{\partial t}+\vec{\nabla} \cdot\left(\rho_{l} \overrightarrow{\boldsymbol{\omega}}\right)=\Omega, \\
\overrightarrow{\boldsymbol{\omega}}=-\frac{k_{\text {soil }}}{\mu_{l}}\left(\vec{\nabla} p+\rho_{l} \overrightarrow{\mathbf{g}}\right), \\
\rho_{l}=\rho_{l}(p), \quad m_{\text {soil }}=m_{\text {soil }}(p), \\
k_{\text {soil }}=k_{\text {soil }}(p), \quad \mu_{l}=\mu_{l}(p) .
\end{gathered}
$$

The equation (16) expresses the mass conservation law, and (17) is set by the Darcy law. The type of parameters functional dependencies on the filtration pressure (18) is assumed to be known.

Subject to the above simplifications and assumptions, the set (16)-(18) will look as follows:

$$
\begin{gathered}
\vec{\nabla} \cdot \overrightarrow{\boldsymbol{\omega}}=0, \\
\overrightarrow{\boldsymbol{\omega}}=-\frac{k_{\text {soil }}}{\mu_{l}}\left(\vec{\nabla} p+\rho_{l} \overrightarrow{\mathbf{g}}\right), \\
\rho_{l}=\text { const }, \quad m_{\text {soil }}=\text { const }, \\
k_{\text {soil }}=\text { const }, \quad \mu_{l}=\text { const. }
\end{gathered}
$$

In this set of equations, the target parameters are velocity vector components and the filtration pressure. The density of liquid, the porosity of the soil layer, the coefficient of its permeability, and the coefficient of dynamic viscosity of liquid are predetermined values. In simulation of the liquid fuel filtration through an anisotropic medium, (20) is substituted with the following equation [9]:

$$
\omega_{i}=-\frac{k_{\text {soil }, i j}}{\mu_{l}}\left(\frac{\partial p}{\partial x_{j}}+\rho_{l} g_{j}\right), \quad i, j=1,2,3 .
$$

After the set of equations (19)-(21) or ((19), (21), (22)) is closed by relevant boundary conditions, it can be solved by the method of finite differences or the method of finite elements [7].

\section{Certain Examples of Practical Application}

Figure 1 shows photographs of actual flames of an isooctane fire set in laboratory conditions and provides an example of results of their numerical simulation which (a) is a photograph of fire without wind; (b) is the isosurface of relative mass fractions of isooctane vapors, corresponding to the highest-rate reaction in the air-and-fuel mixture combusting without wind (stoichiometric mass fraction); (c), (d), and (e) are combustion with a side wind having a speed of $0.05 \mathrm{~m} / \mathrm{s}$. The simulation used a global exothermal single-phase irreversible reaction of isooctane vapors combustion in the air. The isooctane physical and chemical properties used for calculations have been borrowed from [5]. The differences between the geometric parameters of fire obtained in a numerical experiment and measured in a fullscale experiment are presented in Table 1. 
TABLE 1: Comparison of full-scale measurements and results of numerical simulation of an isooctane fire.

\begin{tabular}{|c|c|c|c|}
\hline Parameter & Experiment & Numerical calculation & $\Delta, \%$ \\
\hline Fire length $L, \mathrm{~m}$ & 0.115 & 0.128 & 10.2 \\
\hline Fire width $W$ at a level of $0.01 \mathrm{~m}$ from the liquid surface, $\mathrm{m}$ & 0.032 & 0.026 & 23.1 \\
\hline Angle $\theta$ of fire vertical deviation under a wind of $0.05 \mathrm{~m} / \mathrm{s}$, radian & 0.1651 & 0.2765 & 40.3 \\
\hline
\end{tabular}

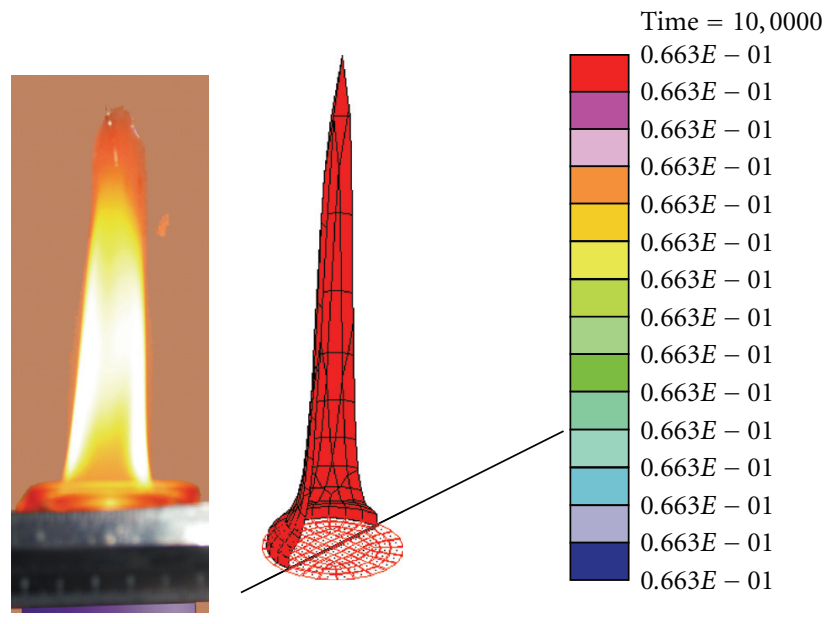

(a)

(b)

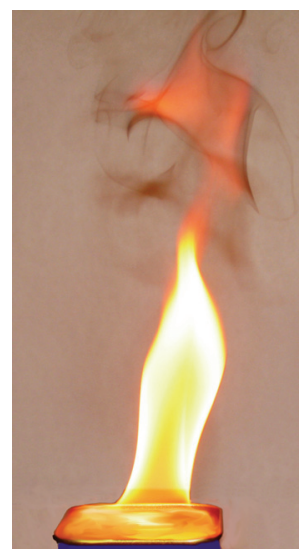

(c)

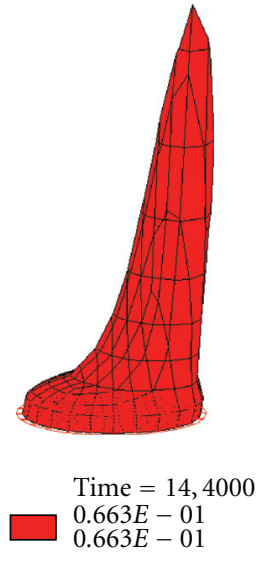

(d)

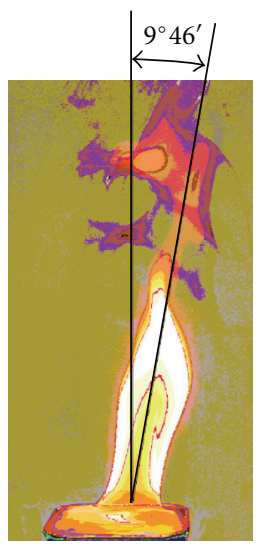

(e)

FIGURE 1: Example simulation of an isooctane pool fire. (a) a photograph of fire without wind; (b) the isosurface of relative mass fractions of isooctane vapors, corresponding to the highest-rate reaction in the air-and-fuel mixture combusting without wind (stoichiometric mass fraction); (c), (d), and (e) are combustion with a side wind having a speed of $0.05 \mathrm{~m} / \mathrm{s}$.

An example of simulation of a full-scale pool fire of spilled gasoline is given in Figure 2.

Also it is necessary to give special priority to comparison of calculated parameters of a pool fire flame according to models (1)-(15) with full-scale measurements of large open pool fire parameters published in [10] and performed for experiments with kerosene and hexane. In this case, the authors paid their attention mostly to kerosene pool fires. It could be explained not only by fuel type used in the tests, but also by the fact that geometrical sizes of combustion source corresponded to large pool fires which are peculiar for oil trunkline networks failures. To confirm the stated above, it is necessary to note that the pool area in tests with kerosene was $12.0 \mathrm{~m}^{2}$ [10]. As for hexane tests, this area was smaller- $4.0 \mathrm{~m}^{2}[10]$.

At full-scale tests with kerosene pool fires, a horizontal cylindrical tank was used to make a controlled pool surface [10]. Flame temperature was measured at four points, corresponding to three different heights on the external wall of the tank. The given points were located at height of $0.72 \mathrm{~m}$, $1.32 \mathrm{~m}$ (two measurements), and $1.92 \mathrm{~m}$ from the pool surface [10]. Plate thermocouples were used for measurements. To increase tests, data adequacy flame temperature was also estimated to be radiometer [10].

A detailed description of the experimental setup [10] along with its basic geometrical dimension as well availability of reference literature containing kerosene thermophysical properties and constants of combustion chemical reaction became a basis for a detailed numerical analysis of test pool fire and comparison of numerical estimations with corresponding results of full-scale measurements. Meanwhile, it is worth mentioning that lack of tests with porous bottom cylindrical tank [10] resulted in the use of models (1)-(15) (i.e., without equations ((19)-(22)) for numerical estimation of the considered pool fires parameters.

At simulation, a period of time from kerosene ignition up to $60 \mathrm{~s}$ of its combustion was considered without extinction system activation (for reference: tests 90 and 91 were simulated from [10]). The atmosphere in the test area considered quiet, wind velocity up to the height of $50 \mathrm{~m}$ from the pool surface was taken to be $1.2 \mathrm{~m} / \mathrm{s}$.

In the full-scale test up to $12 \mathrm{~s}$, an intensive increase of temperature from $293 \mathrm{~K}$ to $\sim 780 \mathrm{~K}$ was seen at all measuring points. Time interval from 12 till $\sim 40 \mathrm{~s}$ was characterized by smooth increase of temperature from $\sim 780 \mathrm{~K}$ to $\sim 1100 \mathrm{~K}$ at all measuring points. After that moment of time up to $60 \mathrm{~s}$, flame temperatures were approximately constant in all cases. A significant effect of temperature change with measuring point height was not observed. In [10], this behavior was explained by the considerable turbulence existing in large fires.

At numerical simulation, curves of temperature increase were qualitatively close to experimental dependences.

At numerical test at the moment of time $12.001 \mathrm{~s}$, the temperature at a height of $0.72 \mathrm{~m}$ was $801 \mathrm{~K}$, at a height of $1.32 \mathrm{~m}$ (measurement 1 ) $-814 \mathrm{~K}$, at a height of $1.32 \mathrm{~m}$ 


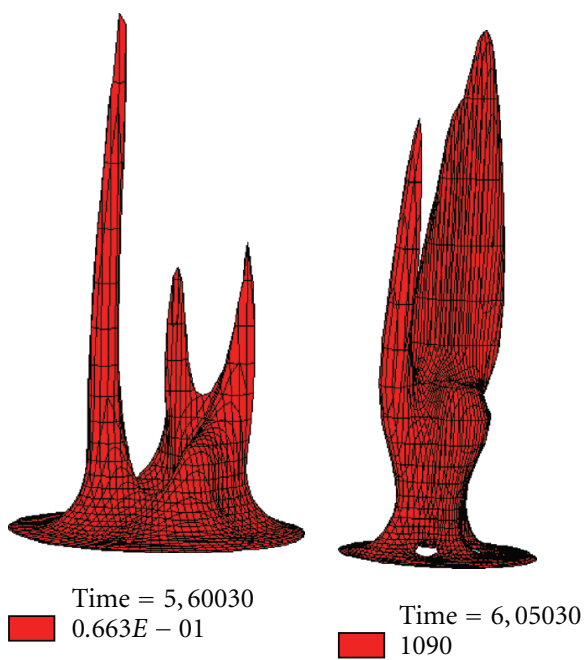

(a)

(b)

FIGURE 2: Example simulation of the time history of a gasoline fire caused by a gas station accident. (a) the isosurface of relative mass fractions of gasoline, corresponding to the highest-rate reaction in the air-and-fuel mixture combusting under a wind load (stoichiometric mass fraction); (b) is the isosurface of the temperature field.

(measurement 2) $-807 \mathrm{~K}$, and at a height of $1.92 \mathrm{~m}-818 \mathrm{~K}$. The arithmetic average value of flame temperatures for all four measuring points was $810 \mathrm{~K}$. Thus, at the first step (ignition), calculated temperature value exceeds measured values at average $30 \mathrm{~K}(\sim 3,8 \%)$. Also it is necessary to note that temperatures differ with the height of measuring points. It can be explained by the fact that at the ignition stage the flow turbulence is not developed enough to equal flame temperature with the height. From now on, the higher calculated temperature values are attributable to the fact that energy consumption needed for measuring thermocouples and their attachment fittings warmup was not taken into account.

At numerical test, at the moment of time $40.001 \mathrm{~s}$, the temperature at a height of $0.72 \mathrm{~m}$ was $1141 \mathrm{~K}$, at a height of $1.32 \mathrm{~m}$ (measurement 1) $-1168 \mathrm{~K}$, at a height of $1.32 \mathrm{~m}$ (measurement 2) $-1159 \mathrm{~K}$, and at a height of $1.92 \mathrm{~m}-1164 \mathrm{~K}$. The arithmetic average value of flame temperatures for all four measuring points was $1158 \mathrm{~K}$. Thus, at the second step (fire development), calculated temperature value exceeds measured values at average $\sim 60 \mathrm{~K}(\sim 5,5 \%)$. However, with the turbulence development the difference between temperature values with the flame height decreases. It corresponds to the results of full-scale tests. At numerical test, the temperature deviation at measuring points located at the same height should be attributed to the peculiar disposition of flame tongues at the moment of time when calculated values were fixed.

Up to the time moment $44.760 \mathrm{~s}$ of the numerical test, quasistationary combustion behavior of fuel vapors was observed. And the average flame temperature of four measuring points decreased to $1142 \mathrm{~K}$. It can be explained by intensive convective heat transfer from combustion source to adjacent atmosphere under condition of developed turbulence.
All comparison results stated above allow us to draw a conclusion that the approach suggested in the article can be successfully applied to simulation of pool fires for accidents prediction and investigation at oil and petroleum derivatives trunkline networks. The size of calculated area at real accidents investigation is recommended to choose no more than several hundreds meters over the surface and upward. Spatial mesh was nonregular. The average mesh spacing at combustion source was about a meter. During the simulation, a time step is recommended to vary from $0,001 \mathrm{~s}$ at ignition and fading of flame to several seconds at quasistationary stage of pool fire. Taking into account the space-time mesh parameters given above, it is natural to talk about nothing else but values of large-scale turbulences (in this case with Reynolds model application (10), (11)). At simulation of trunkline failures when using combustion product in (13), it is recommended to apply general equivalent (integral) coefficients (i.e., $(L=1)$ ) which are responsible for the spectral emission and radiation absorptivity (taking into account soot) [11].

\section{Conclusion}

The above practical method for numerical evaluation of parameters of pool fires caused by trunkline or storage tank failures can be successfully applied in fire and industrial safety analysis of gas, oil, and chemical facilities transmitting or consuming flammable liquids.

At that, results of numerical analysis are used for validation of the geometry and sizes of sanitary protection zones, as well as for investigation of causes and development mechanisms of fires. It should be noted that practical application of this method is well supported by software available in the market. For example, calculation results considered in this article were obtained using such programs as ANSYS/CFX [12] и Star-CD [13].

\section{Nomenclature}

\begin{tabular}{|c|c|}
\hline$A, B:$ & $\begin{array}{l}\text { Specified constants of the diffusion } \\
\text { model of gas combustion }\end{array}$ \\
\hline$C_{i}:$ & $i=\overline{1,4}$ are the specified constants \\
\hline$c_{p}^{\mathrm{vap}}:$ & Specific heat of fuel vapors \\
\hline$c_{\text {rad }}$ & $\begin{array}{l}\text { Velocity of the radiation propagation } \\
\text { in the medium }\end{array}$ \\
\hline$C_{S}:$ & Sutherland constant \\
\hline$C_{\mu}:$ & Specified constant \\
\hline$D_{m}:$ & $\begin{array}{l}\text { Coefficient of binary diffusion in the } \\
\text { mixture }\end{array}$ \\
\hline$D_{\text {vap}}:$ & $\begin{array}{l}\text { Coefficient of fuel vapors binary } \\
\text { diffusion in the atmospheric air }\end{array}$ \\
\hline$e_{m}:$ & $\begin{array}{l}\text { Internal energy of a unit mass of the } \\
m \text { th component }\end{array}$ \\
\hline$f_{\alpha}(\cdot$ & Known semiempirical functions \\
\hline$G:$ & $\begin{array}{l}\text { Dissipation function of turbulent } \\
\text { flow, expressing a thermal equivalent } \\
\text { of mechanical power expendable in } \\
\text { the process of gaseous mixture } \\
\text { deformation due to its viscosity }\end{array}$ \\
\hline
\end{tabular}




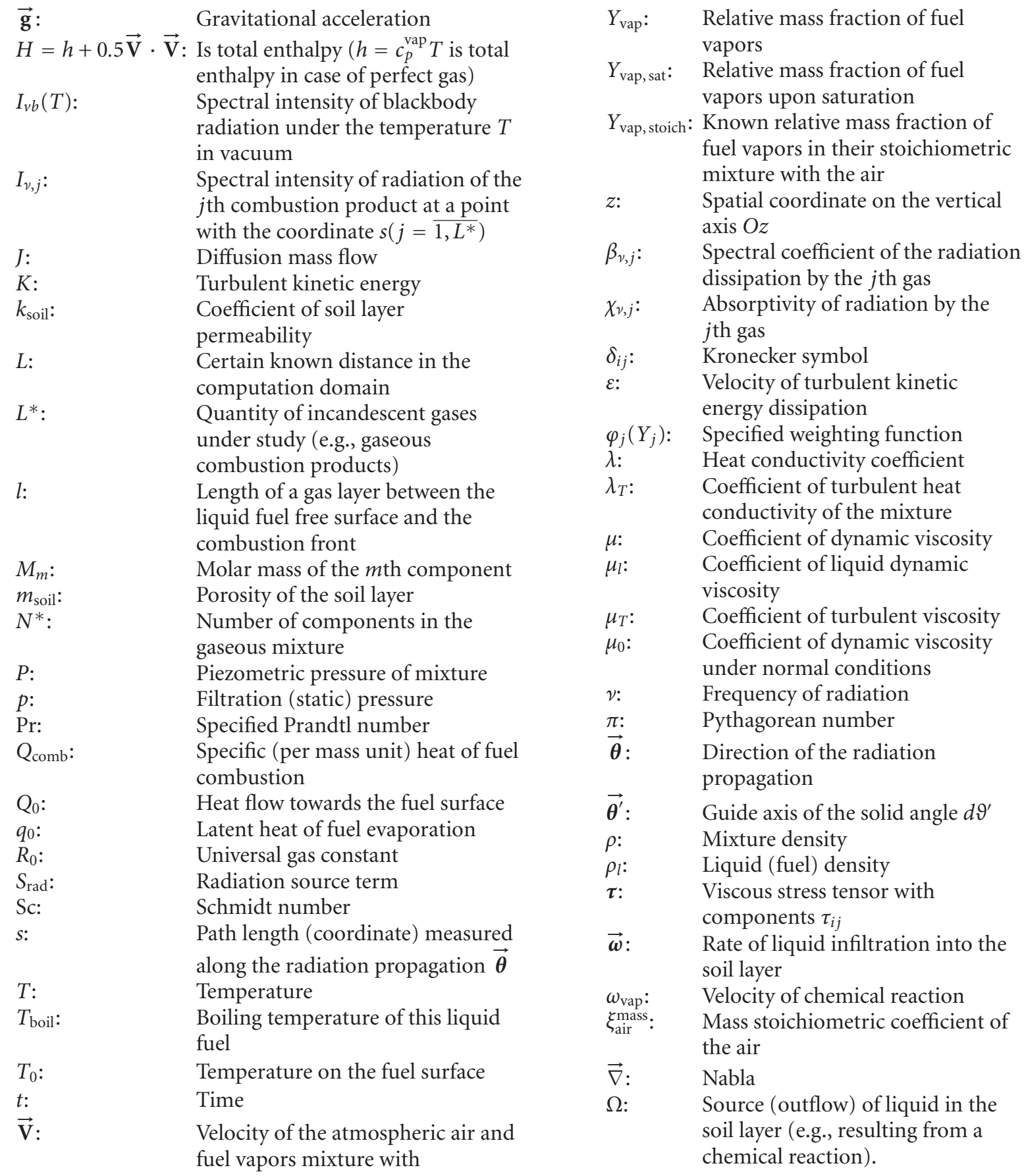

components $u_{1}, u_{2}, u_{3}$

Velocity

$x_{1}, x_{2}, x_{3}$ : Coordinates of the radius-vector of point $\left(x_{3}\right.$ is a coordinate on the vertical axis directed away from the center of the Earth)

$x_{3,0}$ : $\quad$ Fixed coordinate corresponding to the sea level

$Y_{m}=\rho_{m} / \rho: \quad$ Relative mass fraction of the $m$ th component (here, e.g., assignment of $m=1 \equiv$ "vap" corresponds to liquid fuel vapors, $m=2 \equiv$ "air"- to the air (oxidant), $m=3$ 三 "prod"-to combustion products)

\section{References}

[1] V. E. Seleznev and V. V. Aleshin, "Numerical analysis of fire risk at pipeline systems of industrial power facilities," International Journal of Pressure Vessels and Piping, vol. 83, no. 4, pp. 299303, 2006.

[2] D. B. Spalding, Combustion and Mass Transfer, Pergamon Press, London, UK, 1979.

[3] W. M. Kays and M. E. Grawford, Convective Heat and Mass Transfer, McGraw-Hill, New York, NY, USA, 3rd edition, 1993.

[4] J. Warnatz, U. Maas, and R. W. Dibble, Combustion: Physical and Chemical Fundamentals, Modeling and Simulations, Experiments, Pollutant Formation, Springer, New York, NY, USA, 2001. 
[5] V. E. Seleznev, V. V. Aleshin, R. I. Il'kaev, and G. S. Klishin, Numerical Simulation of Gas Pipeline Networks: Theory, Computational Implementation, and Industrial Applications, (Ed. by V. E. Seleznev), KomKniga, Moscow, Russia, 2005.

[6] J. D. Anderson Jr., Computational Fluid Dynamics: The Basics with Applications, McGraw-Hill, New York, NY, USA, 1995.

[7] O. C. Zienkiewicz and R. L. Taylor, The Finite Element Method. Volume 3. Fluid Dynamics, vol. 3, Butterworth-Heinemann, Oxford, UK, 5th edition, 2000.

[8] H. C. Hottel, A. F. Sarofim, and E. J. Fahimian, "The role of scatter in determining the radiative properties of surfaces," Solar Energy, vol. 11, no. 1, pp. 2-13, 1967.

[9] V. N. Schelkachev and B. B. Lapuk, Underground Hydraulics, Regular and Chaotic Dynamics, Izhevsk, Russia, 2001.

[10] E. Planas-Cuchi and J. Casal, "Flame temperature distribution in a pool-fire," Journal of Hazardous Materials, vol. 62, no. 3, pp. 231-241, 1998.

[11] V. A. Akatyev, Fundamentals of Fire and Explosion Safety, RGSU Publishers, Moscow, Russia, 2008.

[12] “ANSYS CFX-Solver, Release 10.0: Theory," ANSYS Inc., 2005.

[13] “Star-CD version 4.10: Methodology," CD-adapco, UK, 2009. 

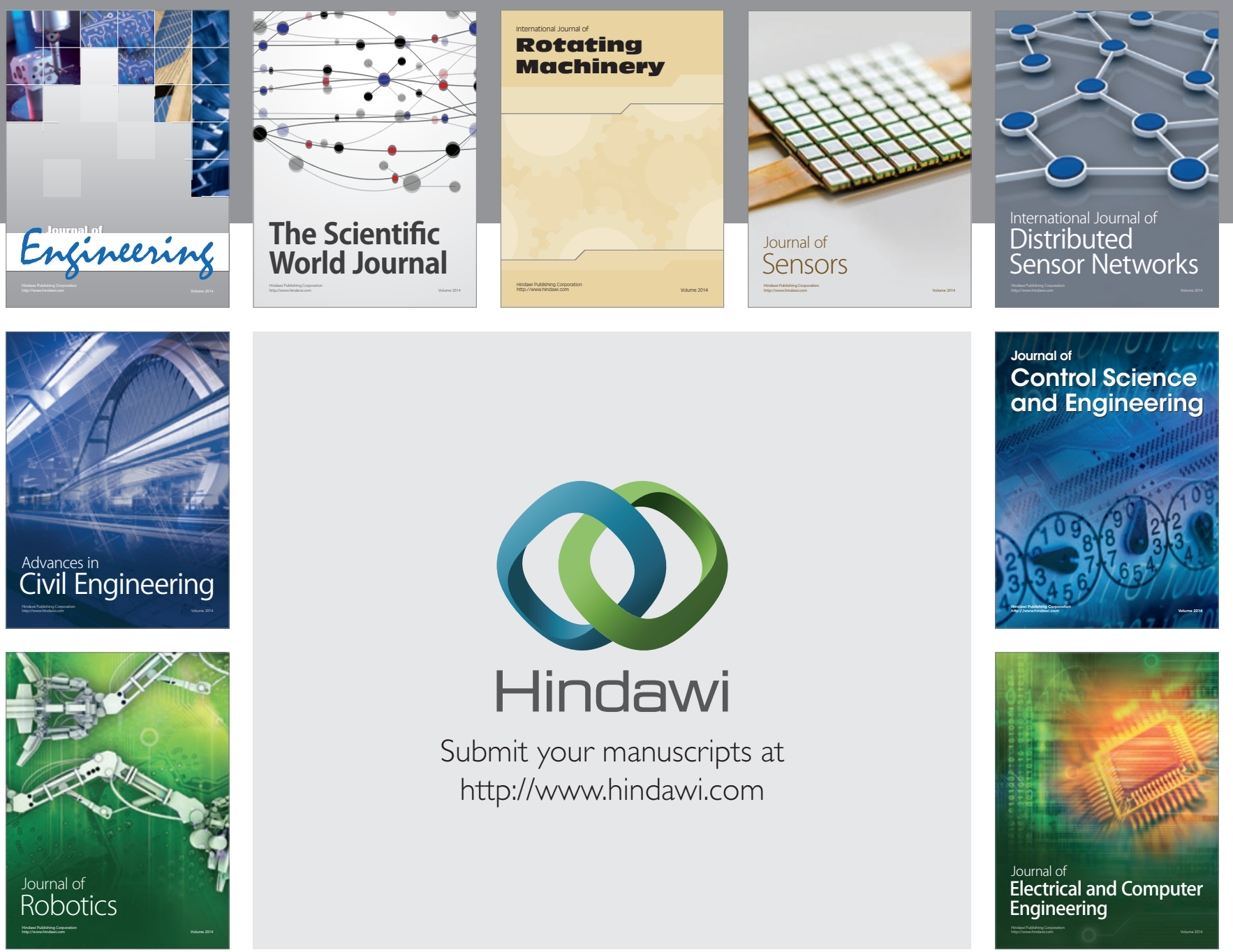

Submit your manuscripts at

http://www.hindawi.com
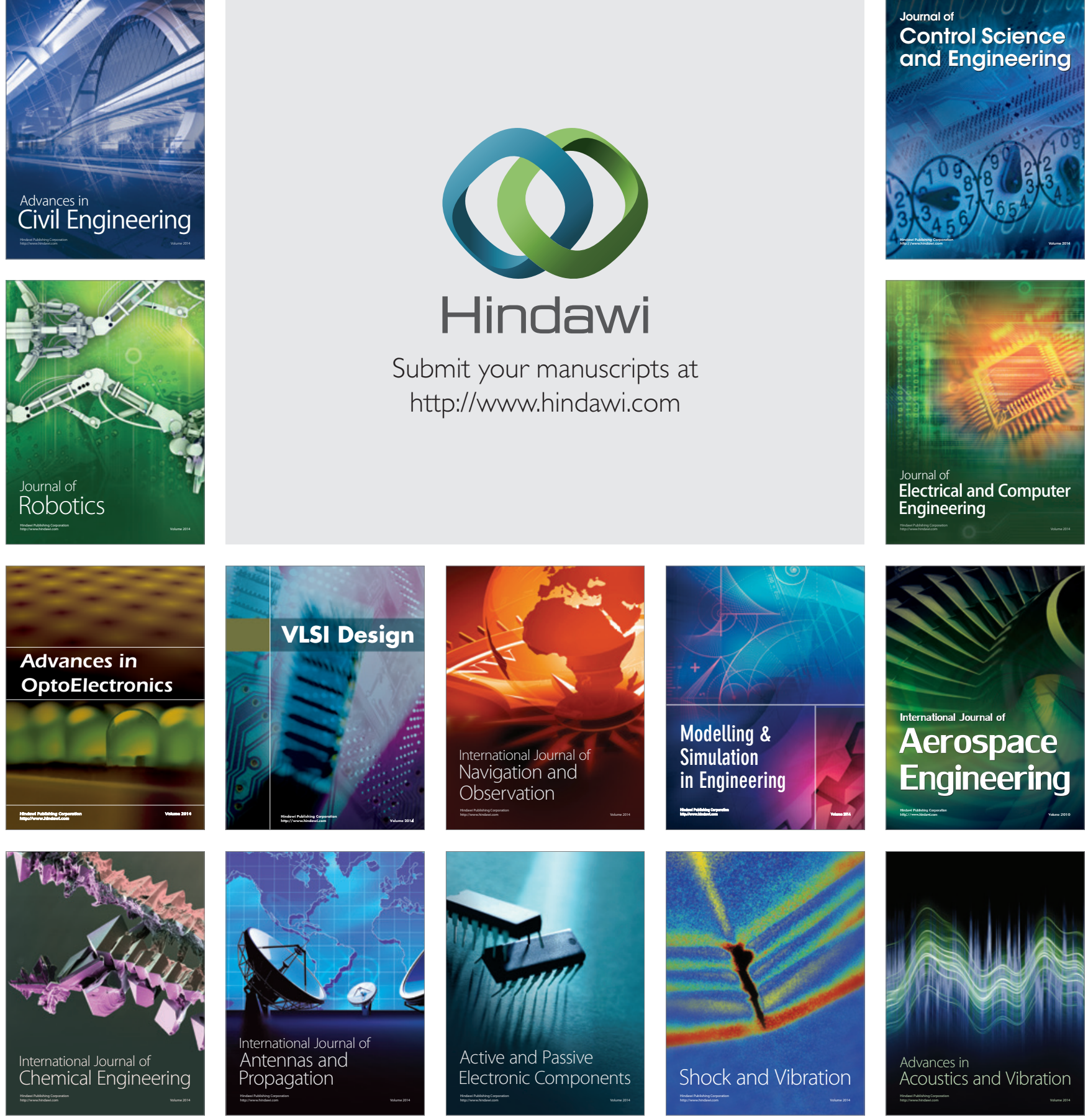